\title{
Mediterranean Diet Mediates the Adverse Effect of Depressive Symptomatology on Short-Term Outcome in Elderly Survivors from an Acute Coronary Event
}

\author{
Christina Chrysohoou, ${ }^{1}$ Catherine Liontou, ${ }^{1}$ Panagiotis Aggelopoulos, ${ }^{1}$ \\ Christina-Maria Kastorini, ${ }^{2}$ Demosthenes Panagiotakos, ${ }^{2}$ Athanasios Aggelis, ${ }^{1}$ \\ Elefterios Tsiamis, ${ }^{1}$ Manolis Vavouranakis, ${ }^{1}$ Christos Pitsavos, ${ }^{1}$ \\ Dimitris Tousoulis, ${ }^{1}$ and Christodoulos Stefanadis ${ }^{1}$ \\ ${ }^{1}$ First Cardiology Clinic, School of Medicine, University of Athens, 46 Paleon Polemiston Street, 16674 Glyfada, Greece \\ ${ }^{2}$ Department of Nutrition-Dietetics, Harokopio University, 70 El Venizelou Av., 17671 Athens, Greece
}

Correspondence should be addressed to Christina Chrysohoou, chrysohoou@usa.net

Received 9 September 2010; Accepted 23 February 2011

Academic Editor: Undurti N. Das

Copyright (C) 2011 Christina Chrysohoou et al. This is an open access article distributed under the Creative Commons Attribution License, which permits unrestricted use, distribution, and reproduction in any medium, provided the original work is properly cited.

\begin{abstract}
Aims. We evaluated the interaction effect between depressive symptoms and dietary habits on 30-day development of cardiovascular disease (CVD) (death or rehospitalization) in elderly, acute coronary syndrome (ACS) survivors. Methods. During 2006-2008, we recorded 277 nonfatal, consecutive ACS admissions ( $75 \pm 6$ years, 70\% males, $70 \%$ had diagnosis of myocardial infarction) with complete 30-day follow-up. Assessment of recent depressive symptoms was based on the CES-D scale. Among sociodemographic, bioclinical, lifestyle characteristics, the MedDietScore that assesses the inherent characteristics of the Mediterranean diet was applied. Results. 22\% of the ACS pts developed a CVD event during the first 30 days ( $14.8 \%$ rehospitalization and 9.4\% death). Patients in the upper tertile of the CES-D scale (i.e., >18) had higher incidence of CVD events as compared with those in the lowest tertile $(21 \%$ versus $8 \%, P=.01)$. Multiple logistic regression analysis revealed that 1 unit increase in CES-D was associated with $4 \%$ higher odds (95\% CI 1.008-1.076, $P=.01$ ) of CVD events; however, when MedDietScore was entered in the model, CES-D lost its significance $(P=.20)$. Conclusion. Short-term depressive symptoms are related to a worsen 30-day prognosis of ACS patients; however, this relationship was mediated by Mediterranean diet adherence.
\end{abstract}

\section{Introduction}

Depression has been recognized as an independent predictor for the development of coronary heart disease (CHD) among healthy and, especially, elderly individuals. Rugulies [1], in a recent meta-analysis, reported that depression increases the risk of myocardial infarction (MI) and coronary death, while that risk is not limited to patients with clinical depression. Similarly, in patients recovering from an acute coronary syndrome (ACS), even the presence of mildly elevated depressive symptoms is associated with a worse prognosis [2]. Additionally, a linear association seems to exist between the occurrence of depressive symptomatology at the time of an MI and the risk of subsequent cardiac morbidity and mortality $[3,4]$.

During the past 30 years, several observational studies and clinical trials have provided scientific evidence that the Mediterranean diet is associated with decreased all-cause mortality and improvements in cardiovascular risk factors levels $[5,6]$. From the late 1960s, Keys and his colleagues from the Seven Countries study underlined the effect of the dietary habits observed in Mediterranean populations on cardiovascular disease risk [7]. De Lorgeril et al., from the Lyon Heart Study, that was a randomized secondary prevention clinical trial of CHD patients, revealed the protective effect of this traditional dietary pattern on cardiac 
complications of the patients within the first 27 months of followup after an acute coronary event [8]. A more recent study in ACS patients illustrated that greater adherence to the Mediterranean diet seems to preserve left ventricular systolic function and is associated with better long-term prognosis of [9].

Although it is generally accepted that depression is independently associated with a worse cardiac prognosis, controversy persists whether this association is a reflection of cardiac disease severity, as some studies have reported significant univariate associations, but have failed to find a significant relationship after adjustment for markers of cardiac disease severity $[10,11]$. Furthermore, other protective lifestyle factors may play a mediator role in the effect of depressive symptomatology on clinical outcome. Among those lifestyle factors, healthy dietary habits due to their anti-inflammatory and antioxidative properties have revealed cardioprotective effect $[8,9]$. Finally, it is well known that the elderly represent the fastest growing segment of the population. In addition, although it is widely adopted that the risk for CVD is an exponential function of age, because of various functional changes observed, the rate of these changes varies greatly among elderly individuals. The relationship between depression and cardiovascular health may also be attributed to lifelong behavioral and lifestyle.

The purpose of this work was to evaluate the role of short-term depressive symptoms in relation to dietary habits on the short-term (i.e., 30-day) clinical outcome of elderly patients who survived ACS.

\section{Methods}

2.1. Study's Sample. From May 2006 to March 2009, 1000 of the 1257 consecutive patients who were hospitalised in our Institution for an ACS (first or recurrent) were enrolled in the study (participation rate 80\%); nonparticipants were patients who denied to provide the requested information or died during the first 48 hours of hospitalization. For the present analysis, 277 elderly (i.e., $>65$ years) patients (75 \pm 6 years, $70 \%$ males, $70 \%$ had a diagnosis of myocardial infarction) that gave the requested information regarding psychological evaluation were studied.

2.2. Bioethics. The study was approved by the Medical Research Ethics Committee of our Institution and was carried out in accordance with the Declaration of Helsinki (1989) of the World Medical Association. All patients were informed about the aims and procedures of the Study and gave their consent to participate.

2.3. Diagnosis of ACS. At entry, a 12-lead electrocardiogram was performed and clinical symptoms were evaluated in all patients by a cardiologist. Moreover, blood tests were performed to detect evidence of myocardial cell death (i.e., Troponin I and the MB fraction of total creatinine phosphokinase $(\mathrm{CPK}))$. As mentioned above, the study's sample included only cases with diagnoses of ACS (i.e., acute myocardial infarction (MI) or unstable angina (UA)). Acute MI was defined according to the latest guidelines
[12] based on ECG findings and the aforementioned blood tests. Additionally, unstable angina was defined by the occurrence of one or more angina episodes, at rest, within the preceding 48-hours, corresponding to class III of the Braunwald classification [13].

2.4. Clinical and Biochemical Measurements. A detailed medical history was recorded, including (i) previous hospitalization for CVD (coronary heart disease or stroke), (ii) history and management of hypertension (defined as systolic or/and diastolic blood pressure $>140 / 90 \mathrm{mmHg}$ or use of antihypertensive medication), (iii) hypercholesterolemia (defined as fasting serum total cholesterol $>200 \mathrm{mg} / \mathrm{dL}$ or use of lipid lowering agents), and (iv) diabetes mellitus (defined as fasting glucose $>125 \mathrm{mg} / \mathrm{dL}$ or use of special treatment), as well as patients' medical family history of CVD. Furthermore the clinical course of all patients during hospitalization was recorded, including coronary angiography results and number of coronary vessels involved, thrombolysis, type of revascularization (primary angioplasty, coronary artery bybass grafting), and time delay from the onset of symptoms to the arrival at the hospital.

In addition to the clinical information, white blood cell counts, uric acid, and brain natriuretic peptide (BNP) were measured at the time of hospital admission. BNP was determined by an enzyme-linked immunosorbent assay (Biomedica, Vienna, Austria), with inter- and intra-assay coefficients of variation being $<5 \%$ and with normal range of $0-100 \mathrm{pg} / \mathrm{mL}$. Total cholesterol, high-density lipoprotein cholesterol, blood glucose, and triglycerides were also measured in all participants at the time of hospital admission using colorimetric enzymic method in a Technicon automatic analyzer RA-1000 (Dade-Behring Marburg GmbH, Marburg, Germany). Low-density lipoprotein cholesterol was calculated using the Friedewald formula: total cholesterol-HDL cholesterol-1/5X (triglycerides). An internal quality control was in place for assessing the validity of cholesterol and triglycerides methods. The intraand interassay coefficients of variation of cholesterol and triglycerides levels did not exceed $4 \%$. Renal function was evaluated using the baseline creatinine clearance rate $(\mathrm{CrCl})$ that was calculated using the Cockcroft-Gault formula: $\mathrm{CrCl}$ $=[[(140-$ age $) \times$ weight $] /(72 \times$ serum creatinine $)]$ for men, while for women, the result of the above equation was multiplied by 0.85 . A Roche/Hitachi Modular analyzer was used (Roche Diagnostics, Manheim, Germany) for all biochemical measurements. The biochemical evaluation was carried out in the same laboratory that followed the criteria of the World Health Organization Lipid Reference Laboratories. Samples were immediately processed for the determination of all biochemical parameters.

2.5. Demographic, Anthropometric, and Lifestyle Characteristics. Sociodemographic characteristics included age and sex. Height and weight were measured to the nearest $0.5 \mathrm{~cm}$ and $100 \mathrm{~g}$, respectively. Body mass index (BMI) was calculated as weight (in kilograms) divided by height (in meters) squared. Obesity was defined as BMI greater than or equal to $30 \mathrm{~kg} / \mathrm{m}^{2}$. To evaluate physical activity status of the patients 
during the past year, a modified version of a self-reported questionnaire provided by the American College of Sports Medicine was used [14]. Based on this questionnaire, the frequency (times per week), duration (in minutes per time) and intensity of sports or occupation-related physical activity were evaluated. Patients who did not report any physical activities were defined as sedentary. Current smokers were defined as those who smoked at least one cigarette per day or have stopped cigarette smoking during the past 12 months. Former smokers were defined as those who had stopped smoking more than one year previously. The rest of the patients were defined as never smokers or rare smokers.

2.6. Dietary Assessment and Evaluation of Adherence to the Mediterranean Diet. The dietary evaluation took place after the third day of hospitalization. Usual dietary intake over the year preceding hospitalization was assessed in all patients by a validated, semiquantitative food frequency questionnaire [15]. The questionnaire included 75 items (i.e., foods and beverages commonly consumed in Greece, as well as dietary habits). Portion sizes were included in the food frequency questionnaire to assist patients to report accurate information and to quantify dietary habits. Alcohol consumption was measured in wineglasses $(100 \mathrm{~mL})$ and quantified by ethanol intake (grams per drink). One wineglass was equal to $12 \mathrm{~g}$ ethanol concentration. The MedDietScore was calculated for each participant in order to evaluate the level of adherence to the Mediterranean diet. In particular, intake of $10 \mathrm{food}$ groups was evaluated (i.e., nonrefined cereals and products, fruits and nuts, vegetables, olive oil, dairy, fish, poultry, potatoes, pulses, red meat or meat products, and eggs), as well as alcohol drinking. The diet score was calculated as follows: for the consumption of items presumed to be close to the Mediterranean pattern (i.e., nonrefined cereals and products, fruits and nuts, vegetables, olive oil, nonfat or low fat dairy, fish, potatoes and pulses), score 0 was assigned when a patient reported no consumption, 1 when reported consumption of 1 to 4 times/month, 2 for 5 to 8 times/month, 3 for 9 to 12 times/month, 4 for 13 to 18 times/month, and 5 for more than 18 times per month. For the consumption of foods presumed to be away from this diet (like meat or meat products, eggs, poultry, and dairy), reverse scores were assigned (i.e., 0 when a patient reported almost daily consumption to 5 for rare or no consumption). Regarding alcohol intake, score 5 was assigned for consumption of less than 3 wineglasses per day, 0 for none or consumption of more than 7 wineglasses per day, and scores of $4,3,2$, and 1 for the consumption of 3 , $4-5,6$, and 7 wineglasses, respectively. This nonmonotonic scoring follows the rationale of the Mediterranean dietary pattern that suggests an intake of $15-30 \mathrm{~g}$ of ethanol per day. Higher values of this diet score indicate greater adherence to the Mediterranean diet (theoretical range 0-55), and have already been associated with CVD risk and markers [16].

2.7. Assessment of Depressive Symptoms. Clinical symptomatology of depression during the past month was determined by a specialised, confidential and weighted questionnaire, which was based on a self-reported depression scale (range 060) developed by Radloff, known as the Center of Epidemiological Studies-Depression scale (CES-D) and validated for the Greek population, by Fountoulakis et al. [17]. The aforementioned scale has been found a reliable and valid measure of depressive symptomatology [18]. Since no accurate cut-offs have been proposed for the Greek population the tertiles of the CES-D score were used to classify the patients. Thus, the participants were divided in three equal size categories (tertiles): (a) 1st tertile (CES-D < 6 ), that represents rare symptoms, (b) 2 nd tertile ( $18<$ CES$\mathrm{D}<26$ ), that represents moderate symptoms, and (c) 3rd tertile (CES-D > 26), that represents more severe symptoms. The questions used for the evaluation of the investigated emotions are listed below: "How often during the past month you..." were bothering by things that usually do not bother you; had poor appetite; feeling that you could not shake off the blues even with help from your family; feeling of being just as good as other people; trouble keeping your mind on what you were doing; feeling that everything you did was an effort; feeling hopeful about your future; thought that your life had been a failure; feeling fearful; having restless sleep; being happy; talking less than usual; feeling lonely; considering people being unfriendly to you; enjoying your life; having crying spells; feeling sad; feeling that people disliked you; could not get "going".

2.8. Follow-Up of the Patients. The lost to follow up rate was $10 \%$ for the 30-days, re-evaluations. Patients lost to followup were considered as missing cases. The follow up of the patients included evaluation for vital status (death from CVD or other cause) and rehospitalization due to acute coronary syndrome (i.e., acute MI or unstable angina as defined above) or other cardiac symptoms (i.e., arrhythmias, stable angina).

2.9. Statistical Analysis. Power analysis (GPower 3.1.1, Germany) showed that the number of enrolled participants $(n=277)$ is adequate to evaluate a 0.05 change in the odds ratio of developing a 30-day adverse CVD event per 1 -unit change in the CES-D score used, achieving statistical power equal to $98 \%$ at $5 \%$ significance level. Normally distributed continuous variables are presented as mean values \pm standard deviation, skewed variables are presented as median and quartiles, while categorical variables are presented as relative (\%) frequencies. Associations between normally distributed continuous variables and group of patients were evaluated through the Student's $t$-test, after controlling for equality of variances (homoscedacity) using the Levene test. The associations between skewed variables (i.e., Troponin I, BNP, glucose, triglycerides) and group of patients were evaluated through the Mann-Whitney test. Associations between categorical variables were evaluated by the use of the chi-squared test, without the correction of continuity. Correlations between continuous variables were evaluated by the use of Pearson's correlation coefficient for the normally distributed and by the Spearman's rho coefficient for the ordinal or skewed variables. Normality was evaluated through the Shapiro-Wilktest. 
The association between the MedDietScore and CES-D score with the occurrence of a CVD event was evaluated using multiple logistic regression analyses, after controlling for socio-demographic, clinical, and biochemical characteristics of the patients. Appropriate tests for goodness-of-fit (i.e., Hosmer-Lemeshow and deviance residuals) were applied in all models. Results are presented as odds ratios and their corresponding 95\% confidence intervals. All statistical calculations will be performed on the SPSS version 18 software (SPSS Inc, Chicago, Il, USA).

\section{Results}

The follow-up evaluation of the patients showed that $22 \%$ had a CVD event during the first 30-days following hospitalization (14.8\% were re-hospitalised and 9.4\% died). Regarding in-hospital mortality, $6 \%$ of the ACS patients died while hospitalized. There were no gender differences between those who developed an adverse cardiac event compared to the rest. In Table 1 clinical, biochemical and lifestyle characteristics of the patients who developed or not an adverse CVD during the 30-day followup are presented.

Moreover, patients in the upper tertile of the CES-D scale (i.e., >18) had higher incidence of 30-day CVD events as compared with those in the lowest tertile $(21 \%$ versus $8 \%$, $P=.01)$; in addition they were more likely to be females (48\% versus $10 \%, P=.001$ ), they had higher prevalence of hypertension ( $88 \%$ versus $63 \%, P=.05)$ and lower current smoking habits ( $27 \%$ versus $20 \%, P=.05)$, while no difference was observed according to diabetes mellitus history, physical activity status, dietary habits, BMI, left ventricular ejection fraction, and previous history of CVD.

In Table 2, clinical, biochemical, and lifestyle characteristics of the patients who developed or not an adverse CVD during the 30-day followup by the tertile of the MedDietScore $(<16,16-19,>19)$ are presented. Among all tertiles of MedDietScore, those patients who presented an adverse cardiac event had lower prevalence of smoking habits, higher plasma BNP levels, higher prevalence of myocardial infarction than unstable angina, lower creatinine clearance levels, more advance age, higher CES-D score, higher diabetes mellitus prevalence, and lower left ventricular ejection fraction.

From the abovementioned results, it seems that a positive relationship between depression status and short-term prognosis of ACS patients exists. However, residual confounding may be present, since several clinical, lifestyle, and behavioural characteristics were associated with adherence to the depression status, as well as the prognosis of the patients. In addition, a significant interaction was observed between CES-D score and MedDietScore $(P=.03)$; thus, the following analysis was stratified by dietary habits class (i.e., away, close, and very close to the Mediterranean diet). Multiadjusted analyses revealed that one-unit increase (1/60) in the Depression scale was associated with $4 \%$ higher odds of developing 30-day adverse cardiac event after discharge, controlling physical activity status, age, gender, BMI, smoking habits, history of hypertension, hypercholesterolemia, diabetes, first event of CVD, and left ventricular ejection fraction. When in the model the MedDietScore was entered, CES-D variable lost its significance, suggesting a strong mediating effect (Table 3).

\section{Discussion}

This study demonstrated that the relationship between depression and CVD development following an ACS in elderly patients is mediated by dietary habits. In particular, previous depression status was initially associated with the development of CVD events; however, when dietary habits were taken into account this relationship was lost. The findings were independent of several potential cofounders such as sex, age, ejection fraction of the left ventricle, body mass index, smoking habits, history of CVD, diabetes mellitus, hypercholesterolemia, hypertension, number of vessel disease, revascularization, and physical activity status.

Previous studies have shown that even minor elevations in depressive symptoms (subthreshold depression, defined to include mood, somatic, and interpersonal symptoms of depression, but not necessarily a diagnosable depressive disorder) significantly increase the risk of incidence of CHD among previously healthy participants or worsen the cardiac prognosis in patients with established CHD [19-21]. The scientific evidence is strongest for patients who have been hospitalized for an ACS, that is, MI or unstable angina [22]. Similarly, according to the GREECS study, which included 2172 patients with ACS, the short-term depressive symptoms are related to more severe disease and a worsen 30-day prognosis of patients hospitalized for ACS [23].

Despite the consistency of the findings in these reviews, it remains unclear why a number of individual studies with robust methodologies have failed to show an adverse effect of depression on mortality especially among the elderly [24, 25]. Furthermore, psychological interventions have failed to show a reduction in mortality, which would be expected if depression caused an increase in mortality [26]. The conflicting results of previous studies may be attributable to the way depression is measured, because different measures may be sensitive to different dimensions of depression, or duration of followup [19]. Furthermore, variation in timing of the assessments and variable attempts to control for the confounding effect of severity of heart disease may be also responsible for the heterogeneity [27]. However, what are less established are the mechanisms responsible for the effect of depression on cardiovascular risk [28]. It has been reported that depression might increase CHD incidence and mortality by promoting factors associated with coronary atherosclerosis. The effect of depressive symptoms may be direct, inducing cardiac ischemia, increasing the risk for cardiac arrhythmias and sudden death, increasing the levels of blood lipids, platelets, and inflammatory factors, as well as indirect by promoting unhealthy behaviours, like cigarette smoking, decreased adherence to medications, physical inactivity, poor, unhealthy diet, and other lifestyle characteristics [29]. Especially for the prognosis of CHD patients, van Melle et al. [30] reported that among myocardial infarction survivors, the rate of depression and the severity of depressive symptoms were significantly related 
TABLE 1: Clinical, biochemical, and lifestyle characteristics of ACS elderly patients $(n=277)$, according to the development of 30-day CVD events.

\begin{tabular}{lccc}
\hline & 30-day CVD event & Free of 30-day CVD event & $P$-value \\
\hline$N,(\%)$ & $61(22)$ & $216(78)$ & .036 \\
Age (yrs) & $76 \pm 6$ & $74 \pm 6$ & .82 \\
Gender (\%male) & 69 & 70 & .197 \\
Body mass index $\left(\mathrm{kg} / \mathrm{m}^{2}\right)$ & $26.7 \pm 5.2$ & $28 \pm 5$ & .001 \\
\hline Hospital admission variables & & & .672 \\
\hline MI (\%) & 85 & 67 & .001 \\
First CVD event (\%) & $52 \%$ & $56 \%$ & .347 \\
Ejection Fraction (\%) & 33 & 44 & .003 \\
Revascularization (\%) & $33 \%$ & $27 \%$ & .56 \\
Creatinine Clearance (mL) & $49.22 \pm 23$ & $60.2 \pm 22$ & .034 \\
Troponin I (ng. mL) & $10.22 \pm 22$ & $8.3 \pm 2.1$ & .005 \\
Glucose levels (mg/dL) & $189.5 \pm 118.7$ & $475 \pm 79.4$ & .007 \\
BNP (pg/mL) & $943 \pm 148$ & & .376 \\
\hline Medical history variables & & $22.68 \pm 12.5$ & .865 \\
\hline CES- scale (0-60) & $34.20 \pm 18.44$ & $59 \%$ & .013 \\
Physical active (\%) & $51 \%$ & $30 \%$ & .901 \\
Smoking current (\%) & $31 \%$ & $53 \%$ & .551 \\
Hypercholesterolaemia (\%) & $71 \%$ & $72 \%$ & .05 \\
Hypertension (\%) & $71 \%$ & $41 \%$ & .064 \\
Diabetes mellitus (\%) & $45 \%$ & $18.1 \pm 4$ & $134 \pm 24$ \\
MedDietScore & $17.5 \pm 4$ & & \\
Systolic blood pressure & $125 \pm 29$ & & \\
\hline
\end{tabular}

TABLE 2: Clinical, biochemical, and lifestyle characteristics of ACS elderly patients $(n=277)$, according to the development of 30-day CVD events and their dietary habits.

\begin{tabular}{|c|c|c|c|c|c|c|c|c|c|}
\hline & \multicolumn{9}{|c|}{ MedDietScore (0-55) } \\
\hline & \multicolumn{2}{|c|}{ Lowest tertile $(<16)$} & \multirow[b]{3}{*}{1} & \multirow{2}{*}{\multicolumn{2}{|c|}{$\begin{array}{l}\text { Middle tertile (16-19) } \\
\text { 30-day CVD event }\end{array}$}} & \multirow{2}{*}{\multicolumn{4}{|c|}{$\begin{array}{c}\text { Upper tertile }(>19) \\
30 \text { day event }\end{array}$}} \\
\hline & \multicolumn{2}{|c|}{ 30-day CVD event } & & & & & & & \\
\hline & Yes & No & & \multicolumn{2}{|c|}{ Yes No } & $P$ & Yes & No & $P$ \\
\hline$N, \%$ & 31 & 78 & & 8 & 75 & & 22 & 63 & \\
\hline Age (yrs) & $77 \pm 6$ & $76 \pm 6$ & .731 & $76 \pm 6$ & $75 \pm 6$ & .707 & $75 \pm 6$ & $75 \pm 5$ & .767 \\
\hline Gender (\%male) & 57 & 59 & .918 & 35 & 38.5 & .632 & 34.5 & 46 & .003 \\
\hline \multicolumn{10}{|l|}{ Hospital admission variables } \\
\hline MI (\%) & 95 & 74 & .001 & 50 & 61 & .745 & 60 & 74 & .458 \\
\hline First CVD event (\%) & 43 & 50 & .664 & 50 & 39 & .745 & 40 & 56 & .425 \\
\hline Ejection Fraction (\%) & 34.5 & 35 & .822 & 57.53 .6 & $56.6 \pm 23$ & .859 & $65 \pm 25$ & $63 \pm 19$ & .817 \\
\hline Revascularization (\%) & 42 & 23 & .229 & 25 & 18 & .807 & 50 & 52 & .925 \\
\hline Creatinine Clearance $(\mathrm{mL})$ & $41.37 \pm 24.94$ & $58.36 \pm 24.74$ & .044 & 50 & 76 & .444 & 80 & 81 & .924 \\
\hline Troponin I (ng·mL) & $6.71 \pm 9.77$ & $10.57 \pm 21.6$ & .415 & $9.5 \pm 13$ & $6.67 \pm 17$ & .751 & $12.4 \pm 27$ & $9.9 \pm 28$ & .819 \\
\hline $\mathrm{BNP}(\mathrm{pg} / \mathrm{mL})$ & $1089 \pm 1043$ & $642 \pm 714$ & .241 & $1460 \pm 1045$ & $629 \pm 870$ & .453 & $744 \pm 440$ & $375 \pm 490$ & .09 \\
\hline \multicolumn{10}{|l|}{ Medical history variables } \\
\hline CES- scale $(0-60)$ & $21 \pm 24$ & $19 \pm 9$ & .947 & $34 \pm 19$ & $25 \pm 15$ & .924 & $41 \pm 20$ & $31 \pm 13$ & .467 \\
\hline $\operatorname{BMI}\left(\mathrm{kg} / \mathrm{m}^{2}\right)$ & $25.4 \pm 3.2$ & $27.5 \pm 3.7$ & .057 & $33 \pm 7.5$ & $26.9 \pm 4$ & .295 & $27 \pm 4$ & $28.5 \pm 9$ & .487 \\
\hline Physical active (\%) & 36 & 47 & .482 & 67 & 61 & .875 & 70 & 70 & .984 \\
\hline Smoking current (\%) & 14 & 32 & .164 & 67 & 33 & .424 & 30 & 30 & .984 \\
\hline Hypercholesterolaemia (\%) & 57 & 53 & .808 & 67 & 55 & .755 & 80 & 65 & .384 \\
\hline Hypertension (\%) & 64 & 62 & .238 & 100 & 69 & .001 & 70 & 70 & .984 \\
\hline Diabetes mellitus (\%) & 43 & 53 & .541 & 67 & 48 & .645 & 60 & 33 & .176 \\
\hline
\end{tabular}


TABLE 3: Results from additive logistic regression models that evaluated the association of CES-D score on the likelihood of developing 30 -day CVD events among $n=277$ elderly ACS survivors.

\begin{tabular}{lcrr}
\hline & OR $(95 \% \mathrm{CI})$ & OR $(95 \% \mathrm{CI})$ & OR $(95 \% \mathrm{CI})$ \\
\hline CES-D (per 1/60 units) & $1.011(0.98-1.046)$ & $1.042(1.008-1.076)$ & $1.025(0.987-1.065)$ \\
First coronary event (Y/N) & $0.909(0.43-1.889)$ & $0.505(0.210-1.217)$ & $0.63(0.234-1.693)$ \\
Age (per 1 year) & $1.015(0.985-1.045)$ & $1.018(0.946-1.096)$ & $0.987(0.908-1.072)$ \\
Male versus female gender & $1.118(0.495-2.524)$ & $0.568(0.224-1.438)$ & $0.731(0.258-2.067)$ \\
Revascularization (Y/N) & - & $2.453(0.97-6.305)$ & $2.477(0.866-7.086)$ \\
Physical activity (Y/N) & - & $1.138(0.476-2.723)$ & $0.868(0.325-2.318)$ \\
Current smoking habits (Y/N) & - & $0.668(0.23-1.941)$ & $0.688(0.215-2.202)$ \\
Body Mass Index (per 1 kg/m $\left.{ }^{2}\right)$ & - & $0.946(0.853-1.048)$ & $0.961(0.866-1.067)$ \\
Hypercholesterolemia (Y/N) & - & $1.869(0.762-4.586)$ & $1.246(0.46-3.37)$ \\
Hypertension (Y/N) & - & $0.983(0.378-2.551)$ & $0.876(0.294-2.604)$ \\
Diabetes mellitus (Y/N) & - & $1.009(0.432-2.354)$ & $1.139(0.428-3.033)$ \\
Ejection fraction (per 1\%) & - & $0.935(0.897-0.975)$ & $0.951(0.903-1.001)$ \\
MedDietScore (per 1/55 units) & - & - & $0.989(0.866-1.130)$ \\
\hline
\end{tabular}

to the severity of left ventricular dysfunction, giving a potential pathophysiological mechanism for the prognosis of patients with depressive symptoms. Moreover, Carney et al. [31] suggested that the dys-regulation of the autonomic nervous system may partially mediate the increased risk for mortality in depressed myocardial infarction patients with frequent ventricular contractions. Furthermore, depression is associated with poor secondary prevention among patients who have had an acute ischemic event. In the large Heart and Soul study [32] of patients with depression and CHD, an association between depressive symptoms and adverse cardiovascular events was largely explained by behavioral factors, especially physical inactivity. It is also illustrated in other studies that unhealthy behaviors accompany depressive symptomatology and can mainly explain the occurrence of adverse cardiovascular events [33].

In the present work, when MedDietScore, that evaluates the level of adherence to a healthy dietary pattern, was included in the analysis, the depression variable lost its significant for the occurrence of adverse cardiac events during the short-term followup. Additionally, the 30-day outcome was inversely related to MedDietScore. The possible cardioprotective effects of Mediterranean diet became widely known for the first time in the 1970s from the Seven Countries Study [7]. Since then, there are numerous indications that adoption of a Mediterranean diet is associated with decreased all-cause mortality and improvements in cardiovascular risk factors levels [34, 35] due to the diet's antithrombotic, anti-inflammatory, and antioxidant effects $[36,37]$. Among those studies, the HALE project revealed the protective effect of Mediterranean type of diet on cardiovascular and total mortality in elderly individuals [38]. It is known that inflammation and oxidative stress accompany the clinical presentation and course of an ACS, affecting infarct size. Infarct size determines postinfarction survival and prognosis, as it is the most important factor of ventricular remodelling process and left ventricular systolic function. Olive oil, which is the main source of fat in the
Mediterranean type of diet, is rich in oleuropein, which has been shown to reduce the infarct size, in experimental models $[39,40]$, and also to protect reperfused myocardium from oxidative damage "in vivo," resulting in more preserved left ventricular systolic function [41]. In a recent study [9], it was revealed that ACS patients that had closer adherence to the Mediterranean diet preserved left ventricular systolic function, while they showed less in-hospital deaths and cardiovascular events during the two-years of followup. These findings were unaltered after controlling for several patient characteristics, like lifestyle behaviours, medical history as well as biological factors at hospital admission. Those cardioprotective mechanisms of Mediterranean diet might modify beneficially the adverse effects of depression symptomatology on cardiovascular system.

\section{Limitations}

The present work has several strengths mentioned above, but also some limitations. For example, only survivors of ACS were studied. Thus, there is no information from patients who died during the first two days of hospitalization (approximately 2\% of the total hospitalizations). For the dietary evaluation, a holistic dietary approach using food patterns (i.e., Mediterranean diet) and not single food approach was used. The rationale of this approach was that overall diet assessment represents a broader picture of food and nutrient consumption of real life. Furthermore, dietary patterns capture the extremes of dietary habits, preempts nutritional confounding, possible effect modification among nutritional variables through the same procedure, and they do not tend to be biased.

\section{Conclusion}

This study highlights the effectiveness of adhering to the Mediterranean diet on cardiac events following ACS, especially for elderly individuals with depression. Regardless of 
any drug treatment prescribed, clinicians should routinely advise patients with acute coronary syndromes to increase their frequency of consumption of Mediterranean foods, as it seems that this type of diet can attenuate the adverse effects of depression on cardiovascular health. Further studies are needed to investigate the most effective way to treat patients suffering from depressive symptoms and convince them to follow more healthy dietary patterns and a healthy lifestyle in general.

\section{Conflict of Interests}

The authors declare that there is no conflict of interests.

\section{Acknowledgments}

The authors would like to present and thank the field investigators of the study: George Metallinos, Athanasios Aggelis, Stefanos Tyrovolas, Andria Christofi, and Vassiliki Sinopoulou, as well as all the participants for the time spent in the interview.

\section{References}

[1] R. Rugulies, "Depression as a predictor for coronary heart disease: a review and meta-analysis," American Journal of Preventive Medicine, vol. 23, no. 1, pp. 51-61, 2002.

[2] N. Frasure-Smith, F. Lesperance, and M. Talajic, "Depression following myocardial infarction: impact on 6-month survival," Journal of the American Medical Association, vol. 270, no. 15, pp. 1819-1825, 1993.

[3] D. E. Bush, R. C. Ziegelstein, M. Tayback et al., "Even minimal symptoms of depression increase mortality risk after acute myocardial infarction," American Journal of Cardiology, vol. 88 , no. 4, pp. 337-341, 2001.

[4] F. Lespérance, N. Frasure-Smith, M. Juneau, and P. Théroux, "Depression and 1-year prognosis in unstable angina," Archives of Internal Medicine, vol. 160, no. 9, pp. 1354-1360, 2000.

[5] G. Assmann, G. de Backer, S. Bagnara et al., "International consensus statement on olive oil and the mediterranean diet: implications for health in Europe," European Journal of Cancer Prevention, vol. 6, no. 5, pp. 418-421, 1997.

[6] D. Trichopoulos and P. Lagiou, "Dietary patterns and mortality," British Journal of Nutrition, vol. 85, no. 2, pp. 133-134, 2001.

[7] A. Keys, A. Menotti, and M. J. Karvonen, "The diet and 15year death rate in the seven countries study," American Journal of Epidemiology, vol. 124, no. 6, pp. 903-915, 1986.

[8] M. de Lorgeril, P. Salen, J. L. Martin, I. Monjaud, J. Delaye, and N. Mamelle, "Mediterranean diet, traditional risk factors, and the rate of cardiovascular complications after myocardial infarction: final report of the Lyon Diet Heart Study," Circulation, vol. 99, no. 6, pp. 779-785, 1999.

[9] C. Chrysohoou, D. B. Panagiotakos, P. Aggelopoulos et al., "The Mediterranean diet contributes to the preservation of left ventricular systolic function and to the long-term favorable prognosis of patients who have had an acute coronary event," American Journal of Clinical Nutrition, vol. 92, no. 1, pp. 4754, 2010.

[10] D. Lane, D. Carroll, C. Ring, D. G. Beevers, and G. Y. H. Lip, "Predictors of attendance at cardiac rehabilitation after myocardial infarction," Journal of Psychosomatic Research, vol. 51, no. 3, pp. 497-501, 2001.

[11] K. H. Ladwig, M. Kieser, J. Konig, G. Breithardt, and M. Borggrefe, "Affective disorders and survival after acute myocardial infarction. Results from the post-infarction late potential study," European Heart Journal, vol. 12, no. 9, pp. 959-964, 1991.

[12] J. S. Alpert, E. Antman, F. Apple et al., "Myocardial infarction redefined-a consensus document of the Joint European Society of Cardiology/American College of Cardiology committee for the redefinition of myocardial infarction," European Heart Journal, vol. 21, no. 18, pp. 1502-1513, 2000.

[13] E. Braunwald, Heart Disease, W.B. Saunders, London, UK, 5th edition, 1997.

[14] R. R. Pate, M. Pratt, S. N. Blair et al., "Physical activity and public health: a recommendation from the Centers for Disease Control and Prevention and the American College of Sports Medicine," Journal of the American Medical Association, vol. 273, no. 5, pp. 402-407, 1995.

[15] C. M. Kastorini, H. J. Milionis, J. A. Goudevenos, and D. B. Panagiotakos, "Modeling the role of dietary habits and eating behaviors' on the development of acute coronary syndrome or stroke: aims, design, and validation properties of a casecontrol study," Cardiology Research and Practice, vol. 2011, Article ID 313948, 7 pages, 2011.

[16] D. B. Panagiotakos, C. Pitsavos, and C. Stefanadis, "Dietary patterns: a Mediterranean diet score and its relation to clinical and biological markers of cardiovascular disease risk," Nutrition, Metabolism and Cardiovascular Diseases, vol. 16, no. 8, pp. 559-568, 2006.

[17] K. Fountoulakis, A. Iacovides, S. Kleanthous et al., "Reliability, validity and psychometric properties of the Greek translation of the Center for Epidemiological Studies-Depression (CESD) Scale," BMC Psychiatry, vol. 1, article 3, 2001.

[18] M. J. Schroevers, R. Sanderman, E. van Sonderen, and A. V. Ranchor, "The evaluation of the Center for Epidemiologic Studies Depression (CES-D) scale: depressed and Positive Affect in cancer patients and healthy reference subjects," Quality of Life Research, vol. 9, no. 9, pp. 1015-1029, 2000.

[19] J. P. van Melle, P. de Jonge, T. A. Spijkerman et al., "Prognostic association of depression following myocardial infarction with mortality and cardiovascular events: a meta-analysis," Psychosomatic Medicine, vol. 66, no. 6, pp. 814-822, 2004.

[20] J. Sundquist, X. Li, S. E. Johansson, and K. Sundquist, "Depression as a predictor of hospitalization due to coronary heart disease," American Journal of Preventive Medicine, vol. 29, no. 5, pp. 428-433, 2005.

[21] J. Barth, M. Schumacher, and C. Herrmann-Lingen, "Depression as a risk factor for mortality in patients with coronary heart disease: a meta-analysis," Psychosomatic Medicine, vol. 66, no. 6, pp. 802-813, 2004.

[22] I. M. Kronish, N. Rieckmann, J. E. Schwartz, D. R. Schwartz, and K. W. Davidson, "Is depression after an acute coronary syndrome simply a marker of known prognostic factors for mortality?" Psychosomatic Medicine, vol. 71, no. 7, pp. 697703, 2009.

[23] C. Pitsavos, D. B. Panagiotakos, S. Arapi et al., "Short-term depressive symptoms and 30-day prognosis of hospitalized patients with Acute Coronary Syndromes; the Greek Study of Acute Coronary Syndromes (GREECS)," Epidemiologia e Psichiatria Sociale, vol. 16, no. 4, pp. 309-315, 2007.

[24] C. M. Dickens, L. McGowan, C. Percival et al., "Lack of a close confidant, but not depression, predicts further cardiac events 
after myocardial infarction," Heart, vol. 90, no. 5, pp. 518-522, 2004.

[25] R. A. Mayou, D. Gill, D. R. Thompson et al., "Depression and anxiety as predictors of outcome after myocardial infarction," Psychosomatic Medicine, vol. 62, no. 2, pp. 212-219, 2000.

[26] K. Rees, P. Bennett, R. West, S. G. Davey, and S. Ebrahim, "Psychological interventions for coronary heart disease," Cochrane Database of Systematic Reviews, no. 2, Article ID CD002902, 2004.

[27] C. F. M. de Leon, "Depression and social support in recovery from myocardial infarction: confounding and confusion," Psychosomatic Medicine, vol. 61, no. 6, pp. 738-739, 1999.

[28] A. Steptoe and D. L. Whitehead, "Depression, stress, and coronary heart disease: the need for more complex models," Heart, vol. 91, no. 4, pp. 419-420, 2005.

[29] D. B. Panagiotakos, C. Pitsavos, C. Chrysohoou et al., "Inflammation, coagulation, and depressive symptomatology in cardiovascular disease-free people; the ATTICA study," European Heart Journal, vol. 25, no. 6, pp. 492-499, 2004.

[30] J. P. van Melle, P. de Jonge, J. Ormel et al., "Relationship between left ventricular dysfunction and depression following myocardial infarction: data from the MIND-IT," European Heart Journal, vol. 26, no. 24, pp. 2650-2656, 2005.

[31] R. M. Carney, W. B. Howells, J. A. Blumenthal et al., "Heart rate turbulence, depression, and survival after acute myocardial infarction," Psychosomatic Medicine, vol. 69, no. 1, pp. 4-9, 2007.

[32] E. J. Martens, P. De Jonge, B. Na, B. E. Cohen, H. Lett, and M. A. Whooley, "Scared to death? Generalized anxiety disorder and cardiovascular events in patients with stable coronary heart disease: the heart and soul study," Archives of General Psychiatry, vol. 67, no. 7, pp. 750-758, 2010.

[33] M. A. Whooley, P. de Jonge, E. Vittinghoff et al., "Depressive symptoms, health behaviors, and risk of cardiovascular events in patients with coronary heart disease," Journal of the American Medical Association, vol. 300, no. 20, pp. 2379-2388, 2008.

[34] C. Pitsavos, D. B. Panagiotakos, C. Chrysohoou et al., "The effect of Mediterranean diet on the risk of the development of acute coronary syndromes in hypercholesterolemic people: a case-control study (CARDIO2000)," Coronary Artery Disease, vol. 13, no. 5, pp. 295-300, 2002.

[35] K. Esposito, R. Marfella, M. Ciotola et al., "Effect of a Mediterranean-style diet on endothelial dysfunction and markers of vascular inflammation in the metabolic syndrome: a randomized trial," Journal of the American Medical Association, vol. 292, no. 12, pp. 1440-1446, 2004.

[36] T. T. Fung, K. M. Rexrode, C. S. Mantzoros, J. E. Manson, W. C. Willett, and F. B. Hu, "Mediterranean diet and incidence of and mortality from coronary heart disease and stroke in women," Circulation, vol. 119, no. 8, pp. 1093-1100, 2009.

[37] C. Chrysohoou, D. B. Panagiotakos, C. Pitsavos, U. N. Das, and C. Stefanadis, "Adherence to the Mediterranean diet attenuates inflammation and coagulation process in healthy adults: the ATTICA study," Journal of the American College of Cardiology, vol. 44, no. 1, pp. 152-158, 2004.

[38] K. T. B. Knoops, L. C. P. G. M. de Groot, D. Kromhout et al., "Mediterranean diet, lifestyle factors, and 10-year mortality in elderly European men and women: the HALE project," Journal of the American Medical Association, vol. 292, no. 12, pp. 1433 1439, 2004.

[39] M. Massaro and R. de Caterina, "Vasculoprotective effects of oleic acid: epidemiological background and direct vascular antiatherogenic properties," Nutrition, Metabolism and Cardiovascular Diseases, vol. 12, no. 1, pp. 42-51, 2002.

[40] I. Andreadou, E. K. Iliodromitis, E. Mikros et al., "The olive constituent oleuropein exhibits anti-ischemic, antioxidative, and hypolipidemic effects in anesthetized rabbits," Journal of Nutrition, vol. 136, no. 8, pp. 2213-2219, 2006.

[41] C. Chrysohoou, C. M. Kastorini, D. Panagiotakos et al., "Exclusive olive oil consumption is associated with lower likelihood of developing left ventricular systolic dysfunction in acute coronary syndrome patients: the hellenic heart failure study," Annals of Nutrition and Metabolism, vol. 56, no. 1, pp. 9-15, 2010. 


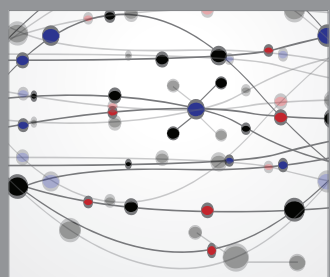

The Scientific World Journal
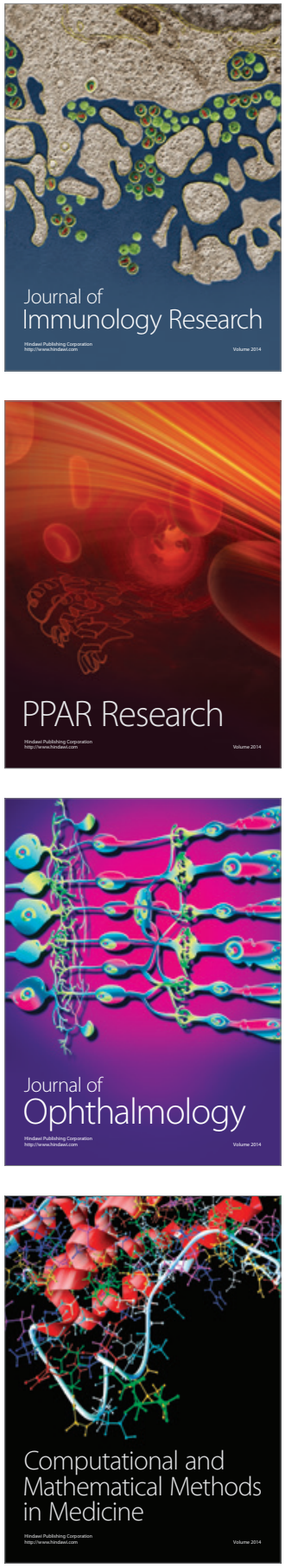

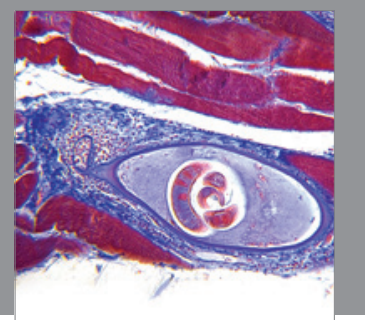

Gastroenterology

Research and Practice
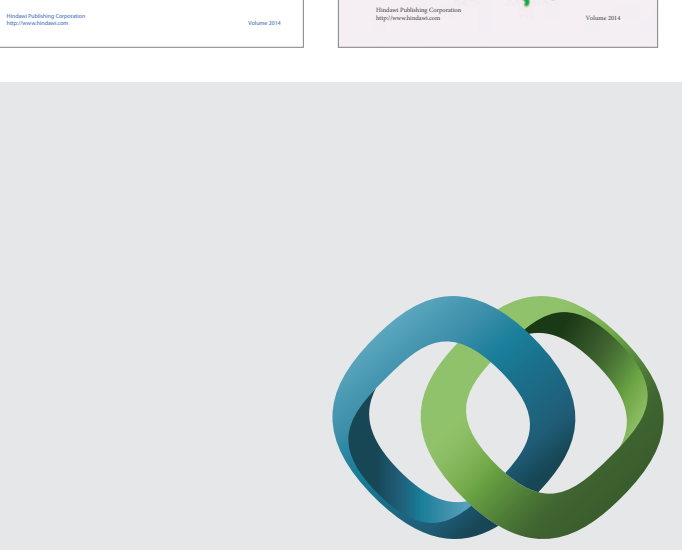

\section{Hindawi}

Submit your manuscripts at

http://www.hindawi.com
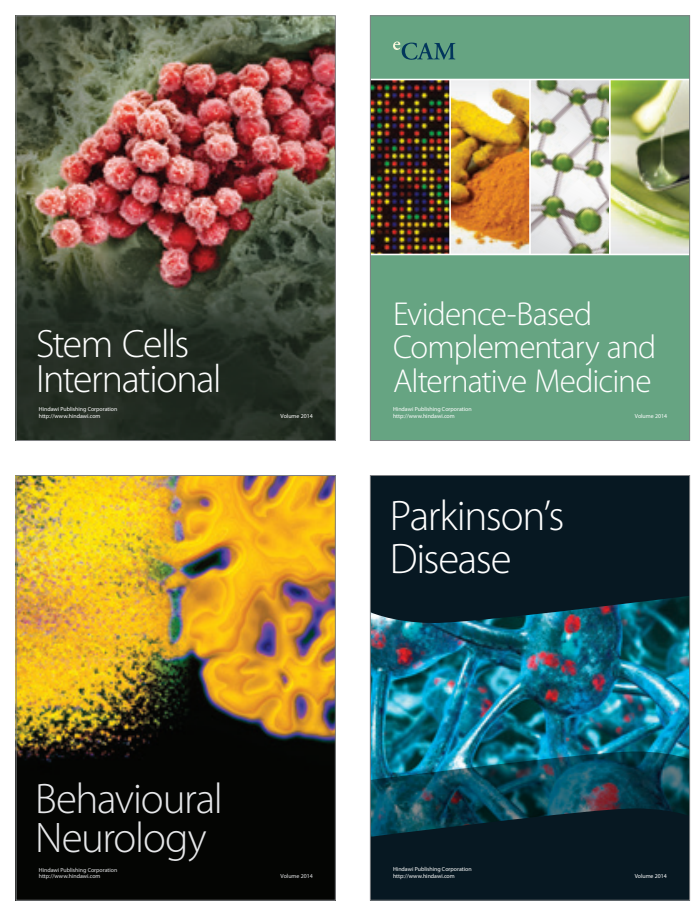

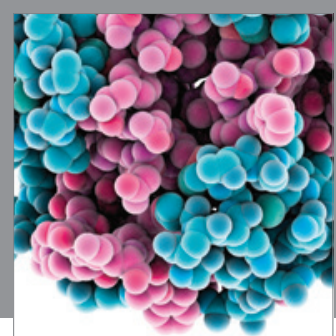

Journal of
Diabetes Research

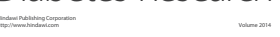

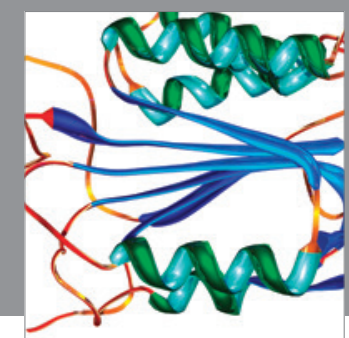

Disease Markers
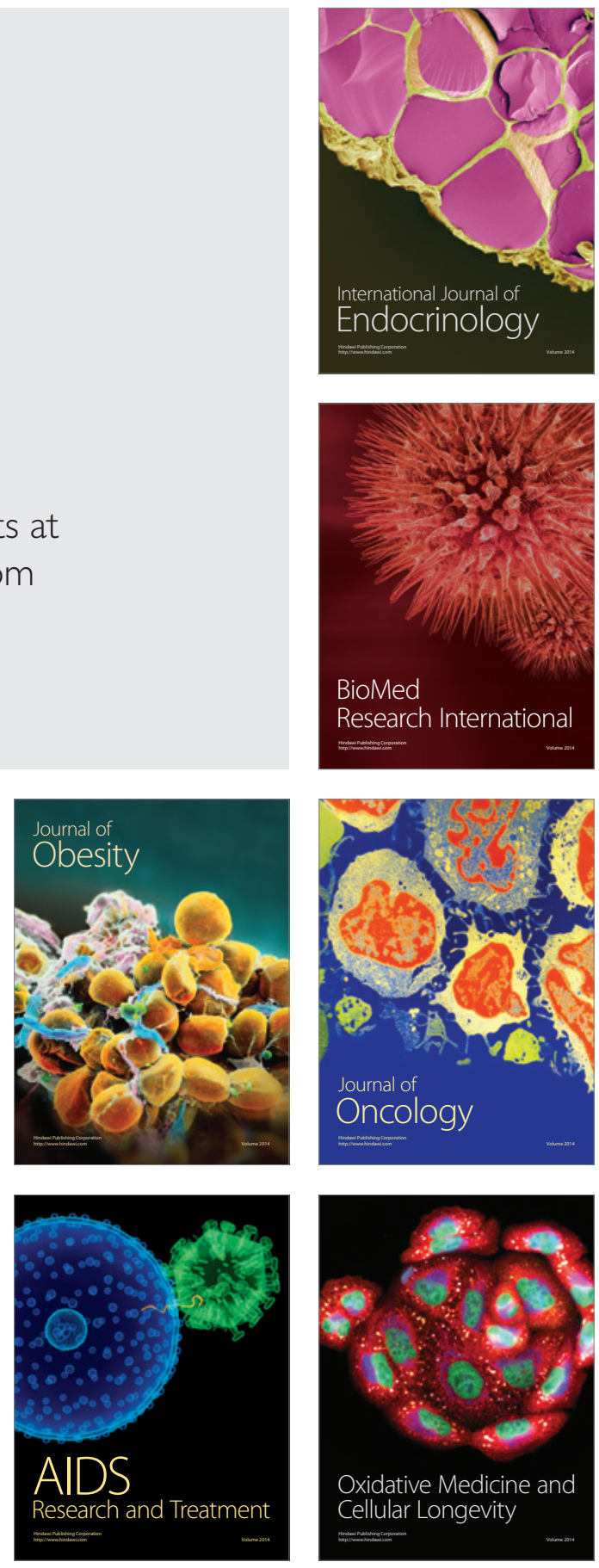